\title{
Analysis and Classification of Strike Rate of a Cricketer: Using Machine Learning Techniques
}

\author{
Soumen Halder* and Arkadip Maitra
}

Department of Computer Science, Ramakrishna Mission Vivekananda Centenary College, Kolkata, West Bengal, India

*Corresponding author: soumen100@rkmvccrahara.org

Received: $24-08-2020$

Revised: 13-11-2020

Accepted: 08-12-2020

\begin{abstract}
Nowadays machine learning and data science, one of the most applied fields of computer science and it has a notable impact on the game of cricket. In this paper we use various ML techniques on cricket. We use 3 different classifiers and one clustering technique. We propose a simple ML based model that classifies data into three different categories that is corresponding to three different formats of cricket. We followed data science life cycle in overall this paper. We compare four ML classifiers in our paper and conclude to the best result. In our data set SVM is best in terms of result and we justify this result with proper statements and figures. And that comparative study is unique to this paper.
\end{abstract}

Keywords: Machine learning (M1), One day International (ODI), Naive Bayes classifiers, SVM (Support vector Machine)

"Where there is data smoke, there is a business fire"

- Thomas Redman

In the $21^{\text {st }}$ century, Data Science and Machine learning are the most trending and emerging fields. You can find and you can apply data science and ML everywhere you wish. Data science is collecting data, processes data,finds insight of data and $\mathrm{ml}$ is used in data science to find that insight. In Supervised learning, you train the machine using data which is well "labeled." It means some data is already tagged with the correct answer. A supervised learning algorithm learns from labeled training data, helps you to predict outcomes for unforeseen data. Unsupervised learning is a machine learning technique, where you

How to cite this article: Halder, S. and Maitra, A. (2020). Analysis and Classification of Strike Rate of a Cricketer: Using Machine Learning Techniques. IJISC., 7(02): 97-107. 
do not need to supervise the model. Instead, you need to allow the model to work on its own to discover information. It mainly deals with the unlabelled data. Unsupervised learning can be more unpredictable compared with other natural learning deep learning and reinforcement learning methods. We in this research use this technology in sports specifically in cricket. In this paper we introduce a ML based Data Science model to analyze cricket data. We try to classify and preded strike rate of a cricketer. We formally use data science life-cycle and modify that to a specific model that can classify and predict future strike rate of a cricketer.

In our paper the main forced point is strike rate and it is defined as; It is a measurement of runs scored per 100 overs faced. A batsman with a high strike rate is considered to score more runs each time they are at bat. strike rate is a more important statistic in shorter games or games with limited overs [4]. In this paper we use the strike of the batsman as a target/dependent variable and we try to analyze and classify this based on a feature space we proposed some new model that easily tells about a batsman. In this competitive world we hope that classification outcome helps a lot. In motivation part we describe the idea in detail and in the methodology part we describe our proposed model and result section we state goodness of our model we also describe exactly which model is best for our data set.

\section{Literature Review}

In this section we will talk about some of the related works that have inspired this paper.

In paper [9] authors Predicting Outcome of Indian Premier League (IPL) Matches Using Classification Based Machine Learning Algorithm they used two machine learning algorithms, Multiple Linear Regression and Random Forests respectively, in order to predict the outcome of Indian premier League matches. In paper [8] Authors states in detailed performance ranking scheme is developed based on Random Forests based Recursive Feature estimation algorithm to rank the players with respect to the other players in fray in IPL 9. In paper [7] authors used IPL sports data and they predicted the various factors that influence the outcome of an Indian Premier League match were identified using a multivariate regression based model and they came to their conclusion. In paper [10] states that shows an analysis for determining the players with higher return of investment potential. These works led to this paper that uses some classification and clustering approaches for predicting the class of players based on some data.

\section{Motivation}

Nowadays data science and ML use all various fields including any such sorts like cricket. In past days some researchers predicted the result of any cricket match specially in limited over cricket. But our recherche is little different from that we are trying to analyze and predict the strike rate of a batsman. The motivating idea is; we try to classify strike rate of cricketer that's how we can tell who is best for which type of cricket like Test, T-20 or ODI based one ml model and farther more we try to predict strike rate (ran getting capability) using regression for future performance. In the recherche data science takes a major role in handling and understanding data and ML coming into picture for prediction and classification purposes.

There have been some related work analysing cricketers and cricket. But, the chief motivation was to use some classification and clustering techniques to group the cricketers unlike the usual methods of multiple regression to predict the grade of a cricketer. 


\section{Methodology}

\section{(a) Analysis and Classification}

In this paper we used an open source dataset that contains cricketer's data. The data contains some commonly used statistics for batsman from various international teams.

Table 1: Snap of the dataset

\begin{tabular}{lllllll}
\hline & Batsman & Total runs & Out & No. of balls & Average & Strike rate \\
\hline 0 & V Kohli & 5426 & 152 & 4111 & 35.697368 & 131.987351 \\
1 & SK Raina & 5386 & 160 & 3916 & 33.662500 & 137.538304 \\
2 & RS Sharma & 4902 & 161 & 3742 & 30.447205 & 130.999466 \\
3 & DA Warner & 4717 & 114 & 3292 & 41.377193 & 143.286756 \\
4 & S Dhawan & 4601 & 137 & 3665 & 33.583942 & 125.538881 \\
\hline
\end{tabular}

Data set contains 6 columns. Before processing data and applying any such ML algorithm we try to approach some basic data science steps that help cleans data and understand data by visualization. We convert 'nan' value and blank space by average value approach.

We take an 'average value approach' to deal with missing values.

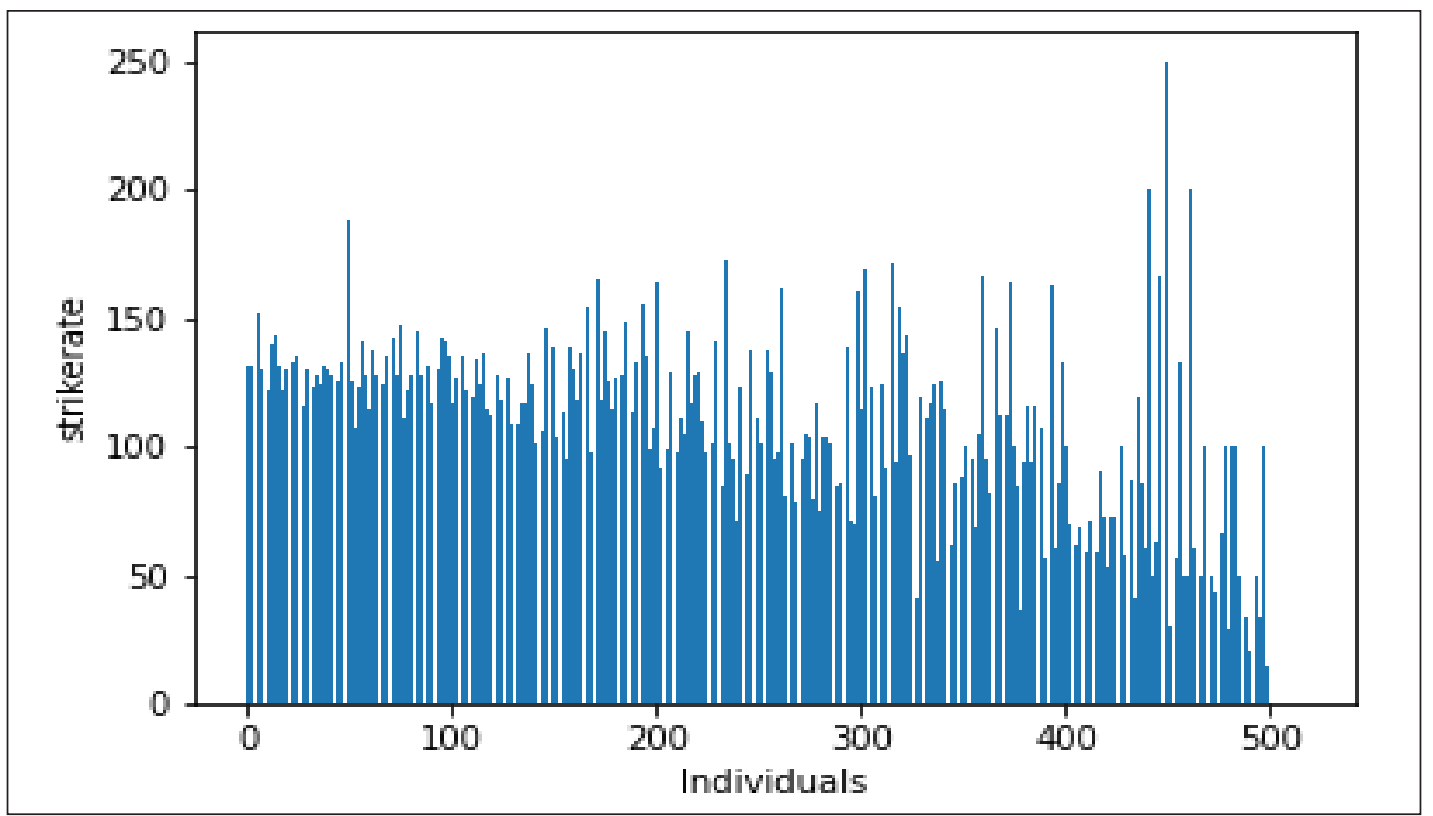

Fig. 1: Bar plot of Strike rate

This is a simple plot to illustrate individuals' strike rate which is our target variable. A bar plot is always good to display the data of a study that involves the comparative changes in things that are numbers based. 


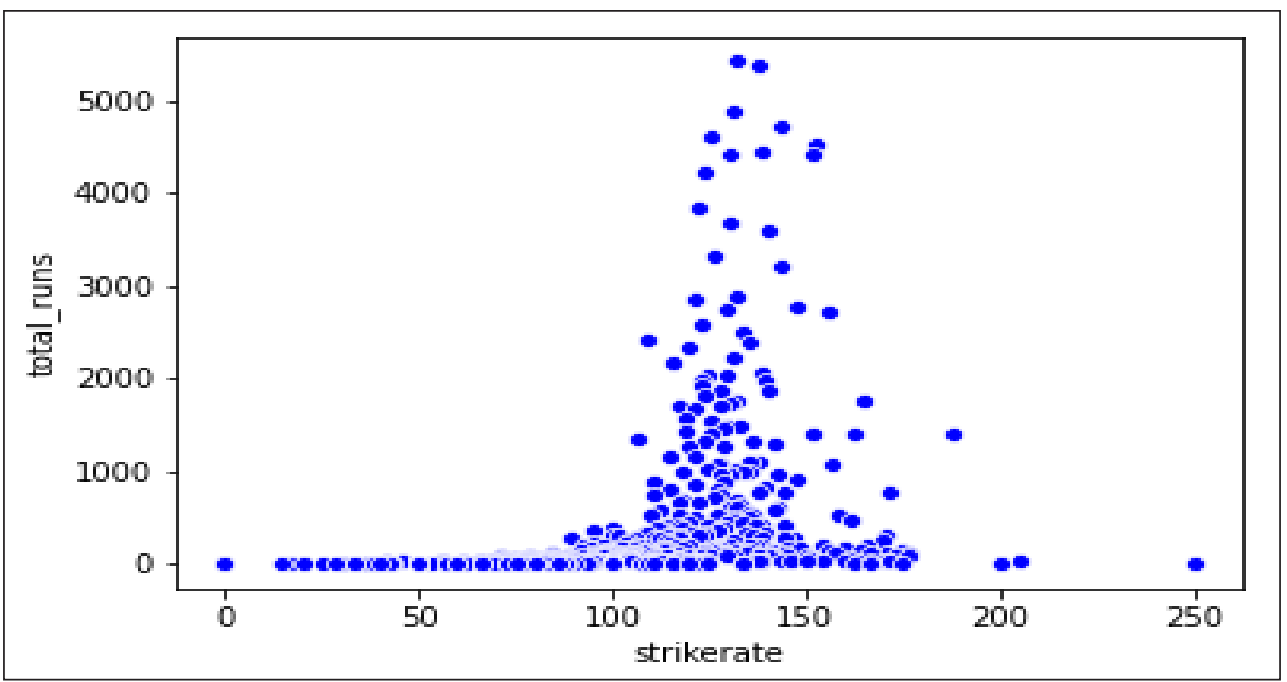

Fig. 2: Relationship between run and Strike rate

This scatter plot very easily depict how much important strike rate and it is related with runs. Now we try to find correlation of data.

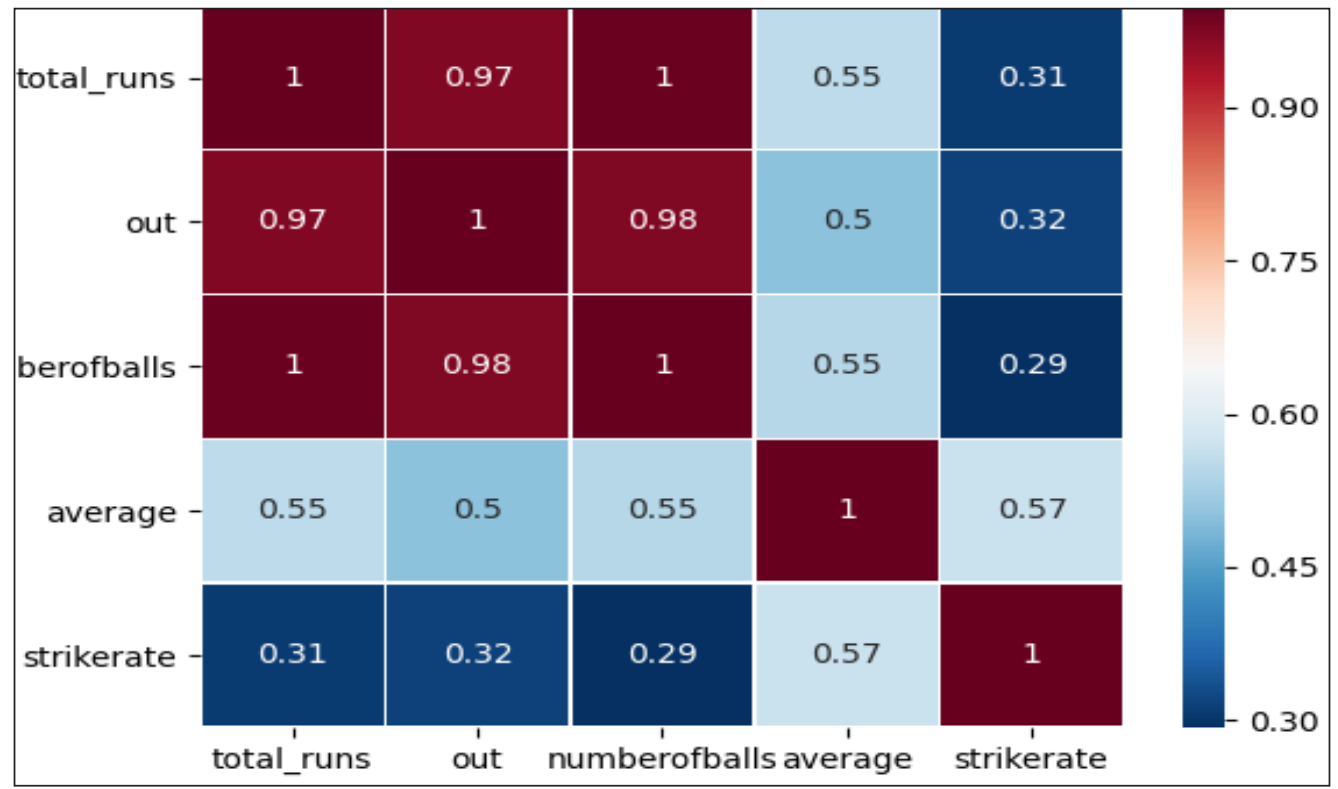

Fig. 3: Correlation Matrix

This correlation matrix allows us to build ml classification models as features are not highly correlated. We consider the threshold of correlation is 0.7. This much analysis helps us apply us to build classification models. We try to build three classes one for Test with low strike rate, for ODI average and high strike rate and T-20 for high and very high strike rate. 


\section{Classification}

Now Machine learning comes into picture as we require to make into data set classification. We use a separate ml classification algorithm. First one is Support Vector Machine [5]. This is very common and mostly used in data science classification algorithms. We use this algorithm as our data set labeled. We also used another supervised classification algorithm that is Naïve Bayes [2].

We consider Class with label 0 indicates players with strike rate below 75, class with label 1 indicates the players with strike rate between 75 and 150 and the class with label 2 indicates the players with strike rate above 150 .

\section{Support Vector Machine (SVM)}

A support vector machine (SVM) is a supervised machine learning model that uses classification algorithms. The goal of the SVM algorithm is to create the best line or decision boundary that can segregate $\mathrm{n}$-dimensional space ( $\mathrm{N}$ - the number of features) into classes so that we can easily put the new data point in the correct category in the future. This best decision boundary is called a hyperplane. In our data we use (total_run, number of bolled_played, out, average) as Independent variable and we consider strick_rate as dependent variable. We consider three different class corresponding to strick_rate we consider The players are divided into three classes in the preprocessing stage.

Steps for Implementing SVM Classifier:

1. Collect data;

2. Clean and fill missing values;

3. Identify the correlation if it .6 or less only then goto step 4 else goto 7 ;

4. Make each class labeled based on you desired result;

5. Implement support vector Machine;

6. Check the accuracy score and generate confusion matrix;

7. Stop.

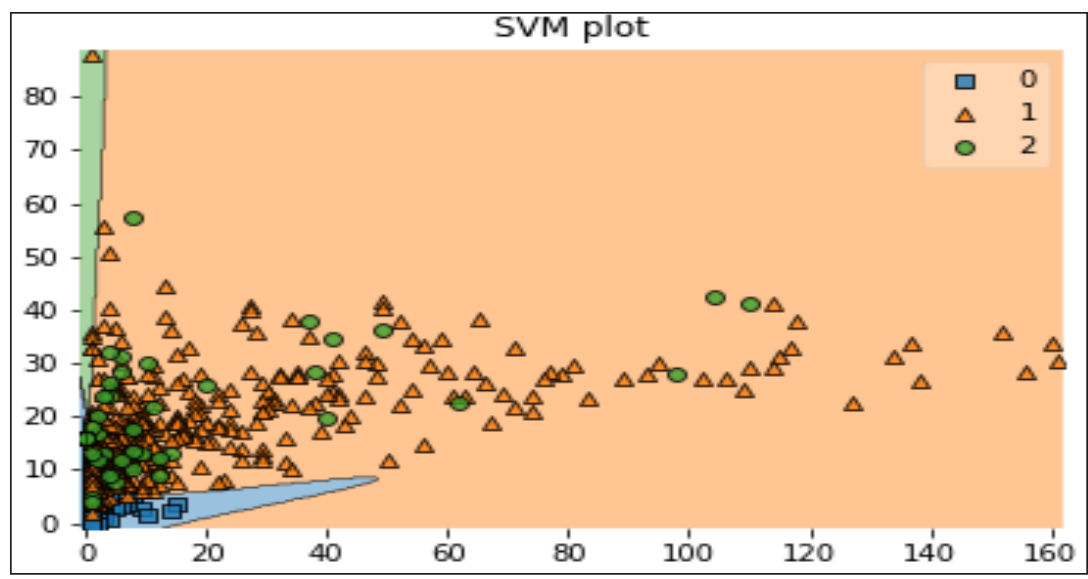

Fig. 4: SVM that applied on our dataset 


\section{Naïve Bayes Classifier}

Naïve Bayes [1] algorithm is a supervised learning algorithm, which is based on Bayes theorem [3] and used for solving classification problems. It works well on any training data including a high-dimensional training dataset. It is a probabilistic classifier, which means it predicts on the basis of the probability of an object. We consider the same tuple that is (total_run,number of bolled_played, out, average) for training purpose. In naive Bayes classifier does not work on normal discrete values we transform that in $(0,1,2)$ based on data point. And after that we simply applied a naive bayes algorithm and considered that result. Steps for Implementing Naïve Bayes Classifier:

\section{Collect data;}

2. Clean and fill missing values;

3. Identify the correlation if it .6 or less only then goto step 4 else goto 8;

4. Transform the data to $(0,1,2)$;

5. Make each class labeled based on you desired result;

6. Implement Naïve Bayes;

7. Check the accuracy score and generate confusion matrix;

8. Stop.

\section{K-Nearest Neighbors}

K Nearest Neighbour could be an easy algorithmic rule that stores all the out there cases and classifies the new information or case supported a similarity live. It's largely accustomed to classify an information purpose supported however its neighbours are classified. ' $\mathrm{K}$ ' in $\mathrm{KNN}$ could be a parameter that refers to the number of nearest neighbours to incorporate within the majority of the pick method.

In the classification setting, the K-nearest neighbor algorithmic rule basically boils right down to forming a majority between the K most similar instances to a given "unseen" observation. Similarity is outlined in keeping with a distance metric between 2 information points. A preferred method for is the euclidean distance technique.

Some of the benefits and drawbacks of KNN:

Simple to implement

Flexible to feature/distance selections

Naturally handles multi-class cases

Can move in follow with enough representative information

Need to confirm the worth of parameter K (number of nearest neighbors)

Computation price is sort of high as a result of we'd like to calculate the space of every question instance to all or any coaching samples.

Storage of information 
There must be a meaningful distance to operate.

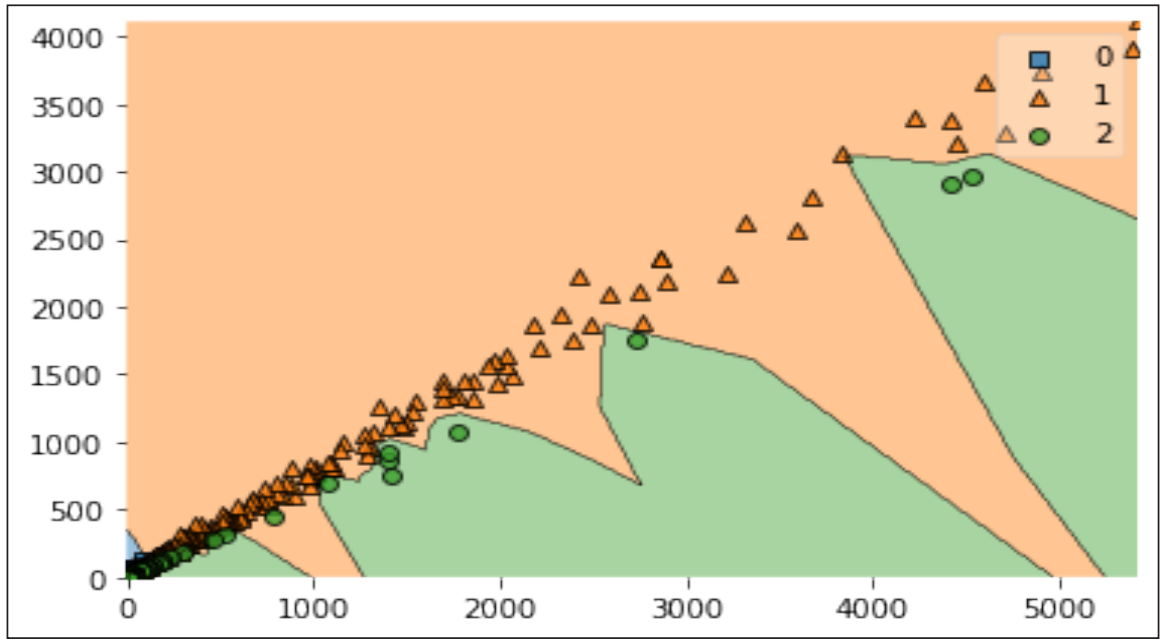

Fig. 5: Output of KNN on the dataset

In the result section we discussed the result of these classifiers with accuracy scores.

\section{Clustering}

Clustering is one of the most common exploratory data analysis techniques used to get an intuition about the structure of the data. It can be defined as the task of identifying subgroups in the data such that data points in the same subgroup are very similar while data points in different clusters are very different. In other words, the target is to try to find homogeneous subgroups within the data such that the data points in each cluster are as similar as possible according to a similarity measure such as euclidean-based distance or correlation-based distance. The decision of which similarity measure is to be used, depends largely on the application.

\section{K Means Algorithm}

$\mathrm{K}$ suggests that is associated in a number repetitious rule that makes an attempt to partition the dataset into $\mathrm{k}$ pre-defined distinct non-overlapping subgroups wherever every datum belongs to only 1 cluster. It tries to form the info points that area unit intra-clustered to be as similar as doable whereas conjointly guaranteeing the clusters area unit as so much as doable. It assigns knowledge points to a cluster specified the add of the square distance between the info points and also the cluster's centre of mass is at the minimum.

The less variation that's given at intervals clusters, the more homogenized the info points area unit at intervals in a similar cluster. Unlike a supervised learning technique, agglomeration is taken into account, we tend to solely need to undertake to research the structure of the info by grouping the info points into distinct subgroups. The target of $\mathrm{k}$ suggests that is to resolve the drawback of Expectation-Maximization. 
The steps to be followed for $\mathrm{k}$ means:

1. Mention the number of clusters K.

2. Initialize centroids by shuffling the dataset and then randomly selecting $\mathrm{k}$ data points for the centroids without replacement.

3. Continue iterating until there is no change to the centroids. i.e assignment of data points to clusters isn't changing.

4. Compute the sum of the squared distance between data points and all centroids.

5. Assign each data point to the closest cluster (centroid).

6. Compute the centroids for the clusters by calculating the average of the all data points that belong to each cluster.

\section{Pros and Cons of $\mathrm{K}$ means that algorithm}

It is simple to implement k-means and determine unknown teams information from complicated data sets.. K-means algorithmic program will simply befit the changes. Its potency depends on the form of the clusters. K-means work well in hyper-spherical clusters. K-means segmentation is linear within the range of knowledge objects therefore increasing execution time. It doesn't take longer in classifying similar characteristics in information like graded algorithms.

It doesn't enable development to associate the best set of clusters and for effective results, you must pick the clusters before. K-means cluster provides varied results on totally different runs of associate algorithmic programs. A random alternative of cluster patterns yields totally different clump results leading to inconsistency. The manner during which information is ordered in building the algorithmic program affects the ultimate results of the info set.

In our case the $\mathrm{k}$ means algorithm was used to cluster the data points into 3 subgroups. The number of clusters are chosen as 3 to resemble the number of classes in the classification methods.

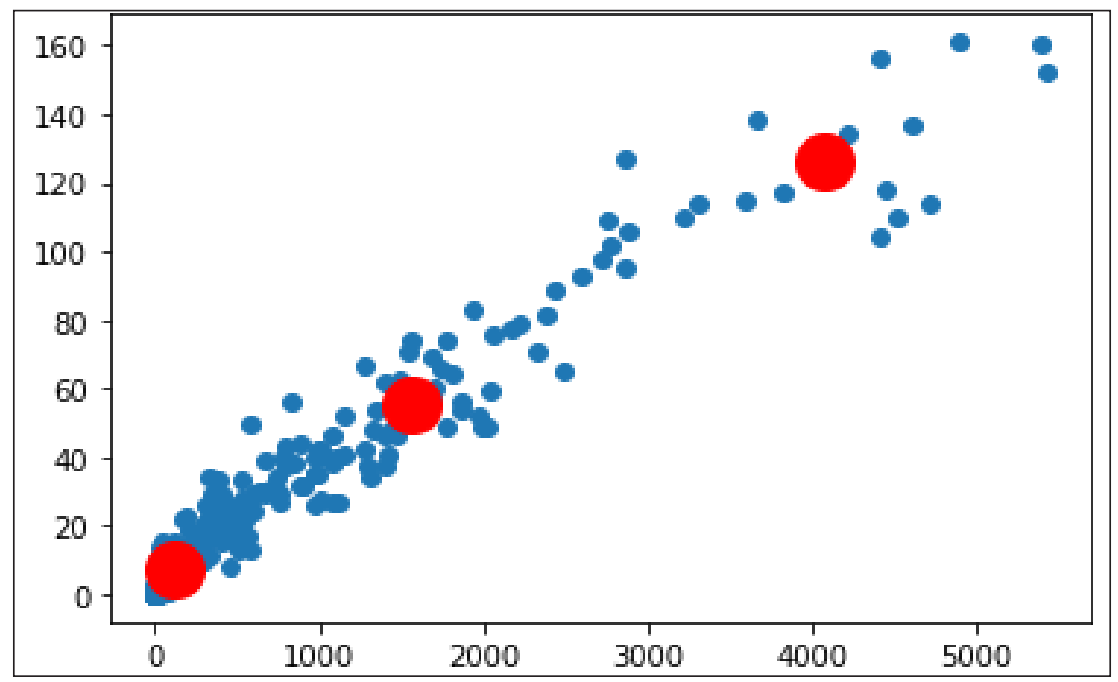

Fig. 6: Output of K means on the dataset 


\section{Workflow of Model}

\section{Proposed Model}

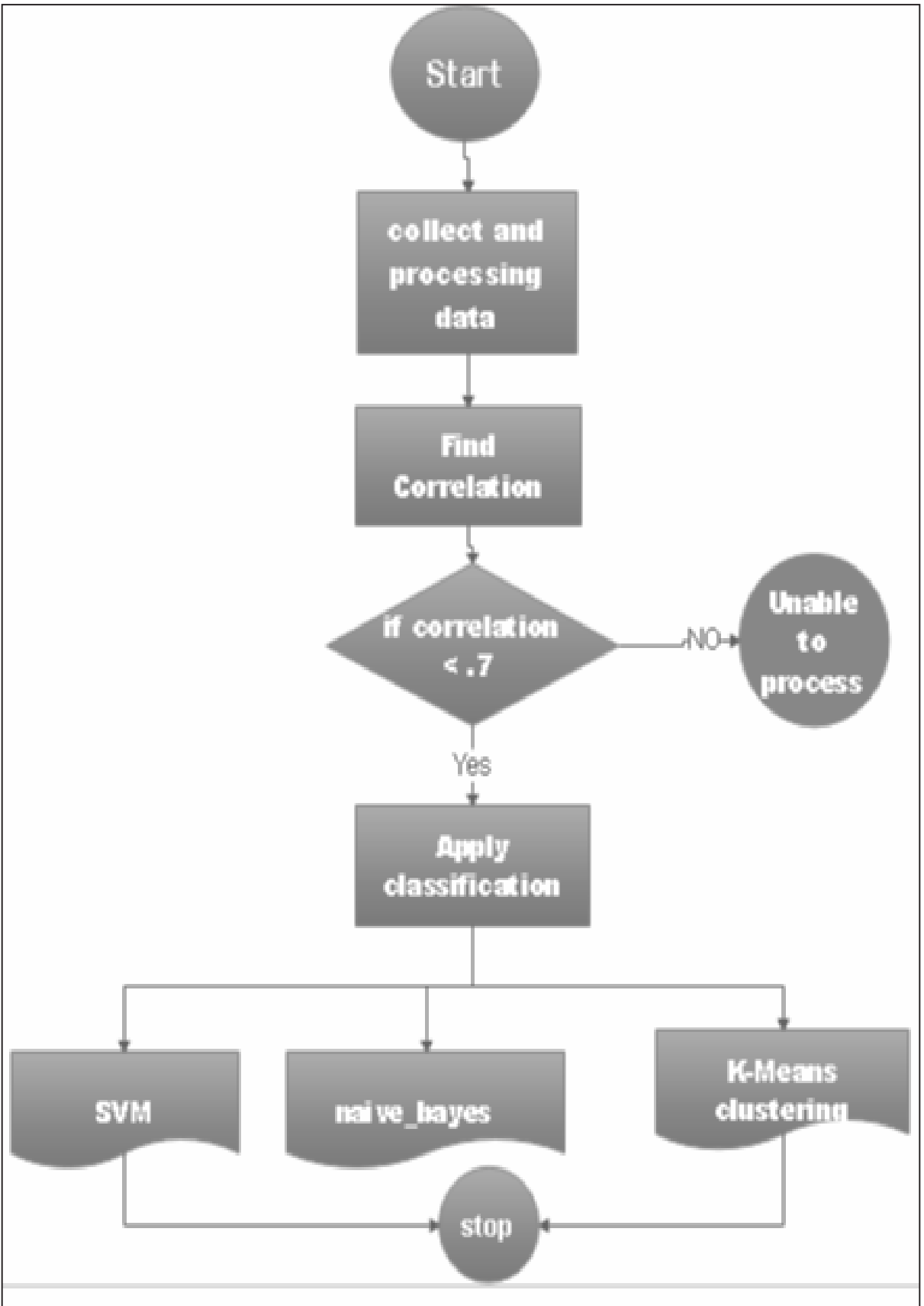

Fig. 7: Data flow model 


\section{RESULTS AND DISCUSSION}

\section{Competitive analysis of the Classification Algorithms}

Table 2: (Result)

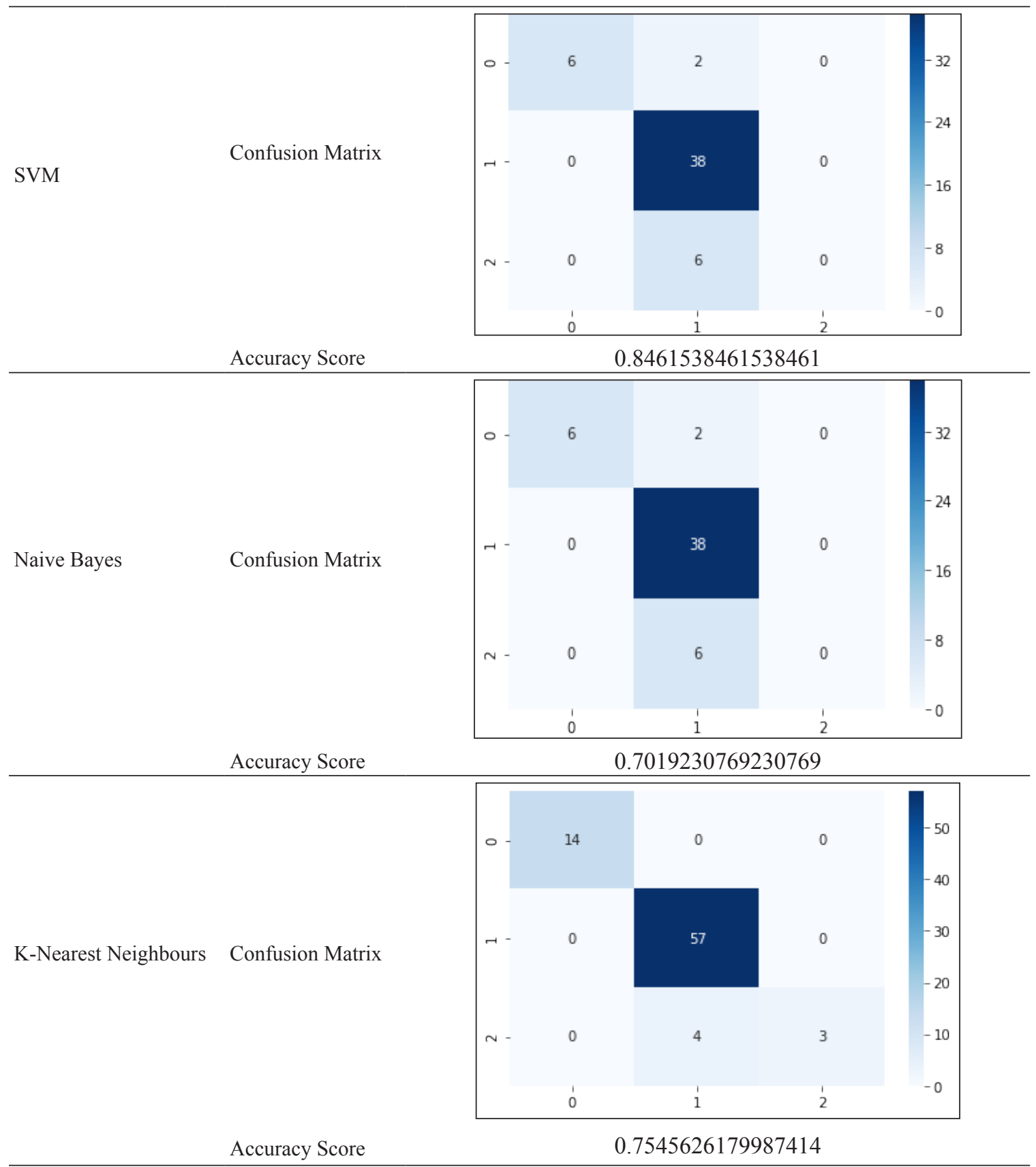


The best measure for understanding any classification is Accuracy score and that accuracy score describes that SVM is most suitable for classification data as that gives us over $80 \%$ accuracy and that is quite good over other applied classifiers.

\section{CONCLUSION}

The application of data science and machine learning techniques is vast in various sports. The analytical and prediction ability of these methods can be a strategic advantage for various sport teams. With availability of data and improvement in computational techniques the application of data science in sports can be extremely effective. This paper tries to apply and compare some classification and a clustering approach for grouping batsman into different classes. This approach can effectively group any batsman based on some factors and can find it's application especially for determining the class of a new player. With more data, the accuracy would definitely get better. Future work might include the application of this paper for other positions like bowler in cricket and also for determining the class of players in other sports.

\section{REFERENCES}

1. Webb, G.I., Keogh, E. and Miikkulainen, R. 2010. Naïve Bayes. Encyclopedia of Machine Learning, 15: $713-714$.

2. Rish, I. 2001. An empirical study of the naive Bayes classifier. In IJCAI 2001 workshop on empirical methods in artificial intelligence, 3(22): 41-46.

3. Swinburne, R. 2004. Bayes'Theorem.

4. https://captaincalculator.com

5. "Comparative study of Different Data Science Classification Algorithms" (ISBN: 978-93-88879-958) chapter-11 of "Information, Communications and Computation Technology (ICCT): The Pillar for Transformation”.

6. Efron, B. 2013. Bayes' theorem in the $21^{\text {st }}$ century. Science, 340(6137): 1177-1178.

7. Lamsal, R. and Choudhary, A. Predicting Outcome of Indian Premier League (IPL) Matches Using Classification Based Machine Learning Algorithm. School of Computer \& Systems Sciences Jawaharlal Nehru University New Delhi, India.

8. Prakash, D. 2016. A New Team Selection Methodology using Machine Learning and Memetic Genetic Algorithm for IPL-9 C. IJEECS, 5(4).

9. Lamsal, R. and Choudhary, A. 2020. Predicting Outcome of Indian Premier League (IPL) Matches Using Machine Learning. School of Computer and Systems Sciences. Jawaharlal Nehru University, New Delhi 110067. arXiv:1809.09813v5 [stat.AP] 21 Sept.

10. Deep Prakash, C. Patvardhan, Sushobhit Singh. 2013. A new Machine Learning based Deep Performance Index for Ranking IPL T20 Cricketers C. Int. J. Comp. Appli., 137(10). 
